\title{
Challenges in identifying Candida auris in hospital clinical laboratories: a need for hospital and public health laboratory collaboration in rapid identification of an emerging pathogen
}

\author{
Amanda J. Durante $\mathrm{PhD}^{1,2}$, Meghan H. Maloney $\mathrm{MPH}^{1}$, Vivian H. Leung $\mathrm{MD}^{1,3}$, Jafar H. Razeq $\mathrm{PhD}^{1,4}$ and \\ David B. Banach $\mathrm{MD}^{2}$ \\ ${ }^{1}$ Connecticut Department of Public Health, Healthcare-Associated Infections/Antimicrobial Resistance Program, Hartford, CT, ${ }^{2}$ University of Connecticut School \\ of Medicine, Farmington, CT, ${ }^{3}$ Epidemic Intelligence Service, Division of Scientific Education and Professional Development, Centers for Disease Control and \\ Prevention, Atlanta, GA and ${ }^{4}$ Katherine A. Kelley State Public Health Laboratory, Rocky Hill, CT
}

To the Editors-Candida auris is an emerging fungus that poses a considerable threat to US healthcare facilities and their patients. Patients exposed to C. auris can develop invasive infection, which can be fatal, ${ }^{1}$ or can become colonized, which poses long-term transmission risks. Once introduced into a healthcare facility, C. auris can spread through contact with affected patients and contaminated surfaces. ${ }^{2}$ The organism can persist in the environment, ${ }^{3}$ and quaternary ammonium disinfectants demonstrate poor activity against it. ${ }^{4}$ Candida auris is often multidrug-resistant, ${ }^{1,4}$ and its detection is challenging because it can be misidentified by some biochemically based identification methods. For example, the API 20 C (bioMerieux, Marcy-l'Etoile, France) can misidentify C. auris as C. sake or Rhodotorula glutinis, and the Vitek 2 (bioMerieux) can misidentify C. auris as C. haemulonii or C. duobushaemulonii. ${ }^{5}$ Rapid and accurate C. auris detection would help hospitals to guide infection control activities intended to prevent the spread of the fungus within and between facilities and to properly plan antifungal treatment. We surveyed laboratories that serve Connecticut's acute-care hospitals to assess their capability to identify C. auris. The information was collected to guide statewide hospital prevention efforts.

During August 2017, we conducted an online survey of C. auris identification and susceptibility testing methods and protocols of hospital-based laboratories. The survey was adapted from an instrument designed by the New Jersey Department of Public Health and was distributed through the Connecticut Laboratory Response Network. Frequency distributions and cross tabulations of survey data were calculated, and results were reviewed by public health and healthcare stakeholders to identify $C$. auris detection gaps. The Centers for Disease Control (CDC) reviewed this study for human subjects protection and deemed it to be a nonresearch study.

Of 23 hospital laboratories, 21 responded to the survey. Of the responding laboratories, 4 contract commercial laboratories for fungal identification, while 17 perform onsite identification. The 17 hospital laboratories that perform onsite fungal identification reported their testing methods. These 17 laboratories serve $80 \%$ of Connecticut's acute-care hospitals. Of these 17 hospital laboratories, 16 (94\%) perform species-level identification for all sterile site isolates. Species-level identification is performed for all

Author for correspondence: Amanda J. Durante, PhD, Immunization Program, Connecticut Department of Public Health, 410 Capitol Avenue, MS \# 11MUN, Hartford, CT, 06134-0308. E-mail: amanda.durante@ct.gov

Cite this article: Durante AJ, et al. (2018). Challenges in identifying Candida auris in hospital clinical laboratories: a need for hospital and public health laboratory collaboration in rapid identification of an emerging pathogen. Infection Control \& Hospital Epidemiology 2018, 39, 1015-1016. doi: 10.1017/ice.2018.133 respiratory Candida isolates at 9 of these laboratories and for all urine Candida isolates at 11 of these laboratories. Only 5 laboratories routinely use matrix-assisted laser desorption/ionization-time of flight (MALDI-TOF) mass spectroscopy for specieslevel Candida identification with a database that can accurately identify $C$. auris, although none use automated $C$. auris alert flags. Furthermore, 11 laboratories routinely use systems for species-level Candida identification that can misidentify or fail to identify C. auris, including the Vitek 2 (6 laboratories), the API 20 C (3 laboratories), the Remel RapID YEAST PLUS (Thermo Fisher Scientific, Lenexa, KS) (1 laboratory), and culture on corn meal agar (1 laboratory). Of these laboratories, 5 have a protocol for the investigation of suspect isolates; however, only 2 have automated alert flags for suspect $C$. auris misidentifications. Only 2 laboratories perform onsite antifungal susceptibility testing on Candida isolates.

Our survey findings demonstrate considerable diversity in Candida identification methods used by Connecticut hospital laboratories and highlight challenges in rapid C. auris detection. Only a minority of laboratories have the capacity to accurately detect $C$. auris, although most use systems for which fungal misidentifications have been characterized (Vitek 2 and API 20 C). This characterization provides an opportunity to implement automated alert flags and protocols for the investigation of potentially misidentified $C$. auris that are not routinely used.

All laboratories that perform species-level identification do so for all sterile-site isolates. However, species-level identification is not performed on all non-sterile-site isolates at some laboratories, which could further limit C. auris detection. Approximately 50\% of US clinical C. auris isolates are identified from nonsterile sites, ${ }^{6}$ although guidance on the optimal strategy for their identification is limited. ${ }^{7}$

These results represent laboratories that serve most of Connecticut's acute-care hospitals. Although our conclusions are strengthened by a high response rate, we recognize the limitation of not having data from commercial laboratories that serve some acute-care hospitals as well as long-term acute-care facilities, where transmission may also occur. ${ }^{2}$ Candida species-level identification methods used in Connecticut hospital laboratories could limit the sensitivity and timeliness of $C$. auris detection, which could delay the implementation of control measures.

The Connecticut Department of Public Health has advised hospitals without appropriate methodology for C. auris species characterization or with isolates that are unidentified or suspect for $C$. auris to contact the health department for guidance. ${ }^{8}$ Additionally, as of November 1, 2017, the Connecticut Public Health Laboratory began offering $C$. auris testing, using polymerase chain reaction and MALDI-TOF, to Connecticut 
healthcare facilities. Challenges of $C$. auris detection emphasize the importance of collaboration between hospitals and the state health department to optimize laboratory capacity for rapid identification of emerging pathogens.

Acknowledgments. The content is solely the responsibility of the authors and do not necessarily represent the official views of the Centers for Disease Control and Prevention or the Department of Health and Human Services.

Financial support. This work was supported by the Centers for Disease Control and Prevention (cooperative agreement no. NU50CK000397).

Potential conflicts of interest. All authors report no conflict of interest relevant to this article.

\section{References}

1. Lockhart SR, Etienne KA, Vallabhaneni S, et al. Simultaneous emergence of multidrug-resistant Candida auris on 3 continents confirmed by whole-genome sequencing and epidemiologic analysis. Clin Infect Dis 2017;64:134-140.
2. Tsay S, Welsh RM, Adams EH, et al. Notes from the field: ongoing transmission of Candida auris in healthcare facilities-United States, June 2016-May 2017. MMWR Morb Mortal Wkly Rep 2017;66:514-515.

3. Welsh RM, Bentz ML, Shams A, et al. Survival, persistence, and isolation of the emerging multidrug-resistant pathogenic yeast Candida auris on a plastic health care surface. J Clin Microbiol 2017;55:2996-3005.

4. Cadnum JL, Shaikh AA, Piedrahita CT, Sankar T. Effectiveness of disinfectants against Candida auris and other Candida species. Infect Control Hosp Epidemiol 2017;38:1240-1243.

5. Mizusawa M., Miller H, Green R, et al. Can multidrug-resistant Candida auris be reliably identified in clinical microbiology laboratories? J Clin Microbiol 2017;55:638-640.

6. Atlanta. Candida auris clinical update-September 2017. Centers for Disease Control and Prevention website. https://www.cdc.gov/fungal/diseases/candidiasis/c-auris-alert-09-17.html. Published 2017. Accessed November 2, 2017.

7. Atlanta. recommendations for identification of Candida auris. Centers for Disease Control and Prevention website. https://www.cdc.gov/fungal/ diseases/candidiasis/recommendations.html. Published 2017. Accessed November 8, 2017.

8. Connecticut Department of Public Health. A case of Candida auris infection at a Connecticut acute care hospital-June 2017. Connecticut Epidemiol 2017;37:9-10.

\title{
Nosocomial impact of prevalent $\beta$-lactamases from the community enterobacteriaceae: what to do when the resistance doesn't go your way
}

\author{
Leandro Reus Rodrigues Perez PhD \\ Hospital Mãe de Deus, Porto Alegre, Brazil
}

To the Editor-Enterobacteriaceae are a common cause of community- and hospital-acquired infections, and they have become increasingly resistant to many classes of antibiotics. ${ }^{1}$ Currently, the emergence of these multidrug-resistant (MDR) organisms has raised global concern, and they require immediate control and prevention. ${ }^{1,2}$

The movement of MDR Enterobacteriaceae into the community in distinct ways (eg, patients with prior hospitalization or genetic determinants of resistance emerging from food or environments) has significant nosocomial impact at the patient admission level and for infection control strategies. ${ }^{2}$

Although studies have found that patients asymptomatically colonized with MDR organisms (eg, extended spectrum $\beta$-lactamase (ESBL) and carbapenemase producers) constitute a reservoir for transmission of the pathogen to others, ${ }^{2,3}$ little is known about the resistance rates among these organisms in the community setting.

Therefore, we conducted a survey to determine the prevalence of MDR Enterobacteriaceae for which MDR was defined as nonsusceptibility to at least 1 agent in 3 or more antimicrobial categories. Urine samples were considered because the recovery of MDR organisms from these samples are most commonly compared with others, especially when enterobacterial species are considered.

Author for correspondence: Leandro Reus Rodrigues Perez, PhD, Microbiology Unit, Hospital Mãe de Deus, 286, José de Alencar street, 90610-000, Porto Alegre RS, Brazil. E-mail: leandro.reus@gmail.com

Cite this article: Perez L, et al. (2018). Nosocomial impact of prevalent $\beta$-lactamases from the community enterobacteriaceae: what to do when the resistance doesn't go your way. Infection Control \& Hospital Epidemiology 2018, 39, 1016-1017. doi: 10.1017/ ice.2018.136
For these MDR isolates, the pattern of antimicrobial susceptibility to a select panel of antibiotics was determined as well.

Enterobacterial isolates were recovered from outpatients between January 1 and December 26, 2016, in Porto Alegre city and its metropolitan area in southern Brazil. Patients with a first MDR-positive urine culture within 48 hours who were admitted from home were deemed to have community-acquired infection and/or colonization.

Antimicrobial susceptibilities to amikacin, ceftriaxone, ciprofloxacin, ertapenem, gentamicin, and trimethoprim/sulfamethoxazole were determined by disk diffusion, and results were interpreted according to the Clinical and Laboratory Standards Institute (CLSI) protocols. ${ }^{4}$ Bacterial identification was performed using the MicroScan WalkAway system (Beckman Coulter, Brea, CA). Resistance mechanisms were detected by phenotypic testing and by gene detection using a previously described polymerase chain reaction (PCR) procedure. ${ }^{5}$

During the study period, a total of 12,193 urinary samples from distinct patients were evaluated. An enterobacterial specie was recovered from 1,885 patients $(15.4 \%)$. Of these 1,885 isolates, $114(6.05 \%)$ were MDR. Among them, 80 isolates ( 80 of $1,885,4.2 \%)$ were ESBL producers, including 65 Escherichia coli, 8 Enterobacter spp, and 7 Klebsiella spp. In addition, 12 isolates $(0.63 \%)$ were non-carbapenemase-producing carbapenemresistant Enterobacteriaceae (nCP-CRE): 7 Enterobacter spp, 4 Klebsiella spp, and 1 Proteus mirabilis. Also, 22 isolates (1.2\%), all Klebsiella pneumoniae isolates, were Klebsiella pneumoniae carbapenemase (KPC) producers. 